\title{
РЕКОМЕНДУЮЩАЯ ПРОГРАММНАЯ СИСТЕМА ОЦЕНИВАНИЯ СОСТАВА ГЕТЕРОГЕННЫХ РОБОТОТЕХНИЧЕСКИХ СРЕДСТВ ДЛЯ ВЫПОЛНЕНИЯ СЕЛЬСКОХОЗЯЙСТВЕННЫХ ОПЕРАЦИЙ
}

\author{
К. Т. Нго ${ }^{\star}$ В. В. Нгуен ${ }^{\star *}$, А. Л. Ронжин ${ }^{\star, * *}$ \\ ${ }^{*}$ Санкт-Петербургский институт информатики и автоматизаиии Российской академии наук \\ ${ }^{* *}$ Санкт-Петербургский государственный университет аэрокосмического приборостроения
}

\author{
Поступила в редакцию 08.09.2019 г.
}

\begin{abstract}
Аннотация. Приведен аналитический обзор работ, направленных на повышение длительности автономной работы БЛА и их обслуживание на сервисных наземных робототехнических платформах, в том числе в области сельскохозяйственной робототехники. При этом в зависимости от размера обрабатываемой территории, необходимой скорости реализации задачи и других факторов зависит количество привлекаемых роботов. Поэтому целью данного исследования являлась разработка математического и программного обеспечения рекомендующей программной системы, обеспечивающей оценивание состава гетерогенных робототехнических средств для выполнения сельскохозяйственных операций. Описан метод многокритериальной оценки количества и состава гетерогенных сельскохозяйственных робототехнических комплексов, необходимых для обработки заданной площади угодья, погодных условий и других аспектов, влияющих на стоимость и скорость выполнения работ. Представлена рекомендующая программная система AgrobotModeling, служащая для моделирования и визуализации процесса взаимодействия нескольких наземных сервисных платформ и беспилотных летательных аппаратов при обработке аграрных объектов. Пользовательский интерфейс, содержащий 4 основных блока, отражающие параметры угодья, платформы и БЛА, а также элементы управления и индикации прошедшего времени, обеспечивает визуализацию и моделирование процесса обработки сельскохозяйственного угодья с различным числом платформ и БЛА.

Ключевые слова: БЛА, робототехническая сервисная платформа, обработка сельскохозяйственного угодия, обслуживание БЛА, взаимодействие гетерогенных роботов.
\end{abstract}

\section{ВВЕДЕНИЕ}

Области применения робототехники в растениеводстве и животноводстве существенно расширяются в последние годы. Совместное использование гетерогенных наземных и воздушных средств увеличивает функциональные и сенсорные возможности робототехнических средств [1-5]. В связи с чем исследование моделей и алгоритмов взаимодействия гетерогенных сельскохозяйственных робототехнических комплексов является актуальным научным направлением, ориентированным в том числе на решение проблемы увеличения времени функциони-

() Нго К. Т., Нгуен В. В., Ронжин А. Л., 2019 рования беспилотных летательных аппаратов (БЛА) на сельскохозяйственных полях, что в конечном итоге будет способствовать сокращению сроков и стоимости обработки сельскохозяйственных объектов за счет автоматизации и применения роботизированных комплексов [6-11].

\section{АНАЛИЗ РЕЗУЛЬТАТОВ ПРЕДШЕСТВУЮЩИХ РАБОТ}

Для повышения автономности беспилотного летательного аппарата требуется, в том числе подзарядка его источника энергии и пополнение других расходных материалов на основе автоматизированных систем перезарядки. В работе [12] разработаны два типа 
автоматических систем перезарядки БЛА на наземной платформе с зарядкой аккумулятора и с заменой на новый. Системы с заменого аккумулятора может значительно сократить время подготовки БЛА для нового полета и увеличить общее число БЛА, находящихся одновременно в автономной миссии. Система подзарядки имеет более низкую стоимость по сравнению с системой замены аккумулятора за счет минимизации механических узлов конструкции.

В работе [13] обосновывается, что поиск оптимальной траектории движения группы БЛА с учетом периодического обслуживания систем энергопитания на сопровождающей группе наземных мобильных заряжающих станций при выполнении некоторой миссии является NP-полной задачей, и для ее решения предлагается ряд модификаций методов линейного программирования и эвристических подходов.

В работе [14] БЛА рассматриваются как транспортные средства, оценивается стоимость их массового использования с учетом обслуживания на стационарных дозаправочных станциях, с учетом очередности обработки БЛА и динамических задержек в прилете БЛА.

В работе [15] исследуются различные подходы к управлению двигателями БЛА, с целью оптимизации энергопотребления. Также выделяются три уровня управления БЛА, на которых можно оптимизировать энергетические затраты: 1) высший - расчет траекторий движения с учетом облета препятствий и минимизацией времени (или потребляемой энергии); 2) средний - расчет кинематических и динамических моделей движения БЛА по заданной траектории с необходимой скоростью; 3) низший - расчет параметров контролеров и преобразователей напряжения, источников тока для поддержания необходимой скорости вращения роторов БЛА. Для низшего уровня управления сравнивается методы Ляпунова, линейной алгебры, ПИД регулятор. При этом отмечается, что первые методы позволяют снизить энергопотребление, но требуют длительного процесса настройки, а ПИД регулятор остается по-преж- нему наиболее простым и относительно эффективным подходом.

В работе [16] также проведен обзор подходов к сокращению энергопотребления систем управления моторами БЛА. Проведен анализ эффективности различных источников тока, а также энергопотребления БЛА на различных этапах функционирования: взлет, набор высоты, перелет, снижение высоты, посадка. Наиболее энергозатратные этапы при наборе высоты и посадке (маневрирование при позиционировании на заданную посадочную площадку) требуют особого внимания и оптимизации алгоритмов управления. Приводятся преимущества и недостатки эвристических, интеллектуальных (нечеткая логика, искусственные нейронные сети и др.) и оптимизационных (динамическое программирование и др.) методов.

По результатам проведенного аналитического обзора работа по тематике исследования необходимо отметить, что задача повышения длительности автономной работы БЛА и их обслуживания на сервисных наземных робототехнических платформах является актуальной задачей, в том числе в области сельскохозяйственной робототехники. При этом в зависимости от размера обрабатываемой территории, необходимой скорости реализации задачи и других факторов зависит объем привлекаемых роботов. Поэтому целью данного исследования являлась разработка математического и программного обеспечения рекомендующей программной системы, обеспечивающей оценивание состава гетерогенных робототехнических средств для выполнения сельскохозяйственных операций.

\section{МАТЕРИАЛЫ И МЕТОДЫ}

Для оценивания необходимого количества техники для обработки сельскохозяйственного угодья был разработан многокритериальный метод с использованием трех основных критериев: 1) суммарное время обработки $t^{\text {sum }}$; 2) суммарная израсходованная энергия $e^{\text {sum }}$; 3) суммарная стоимость задействованной техники $c^{\text {sum }}$. Для моделирования и определения оптимального количества тех- 
Рекомендующая программная система оценивания состава гетерогенных робототехнических ...

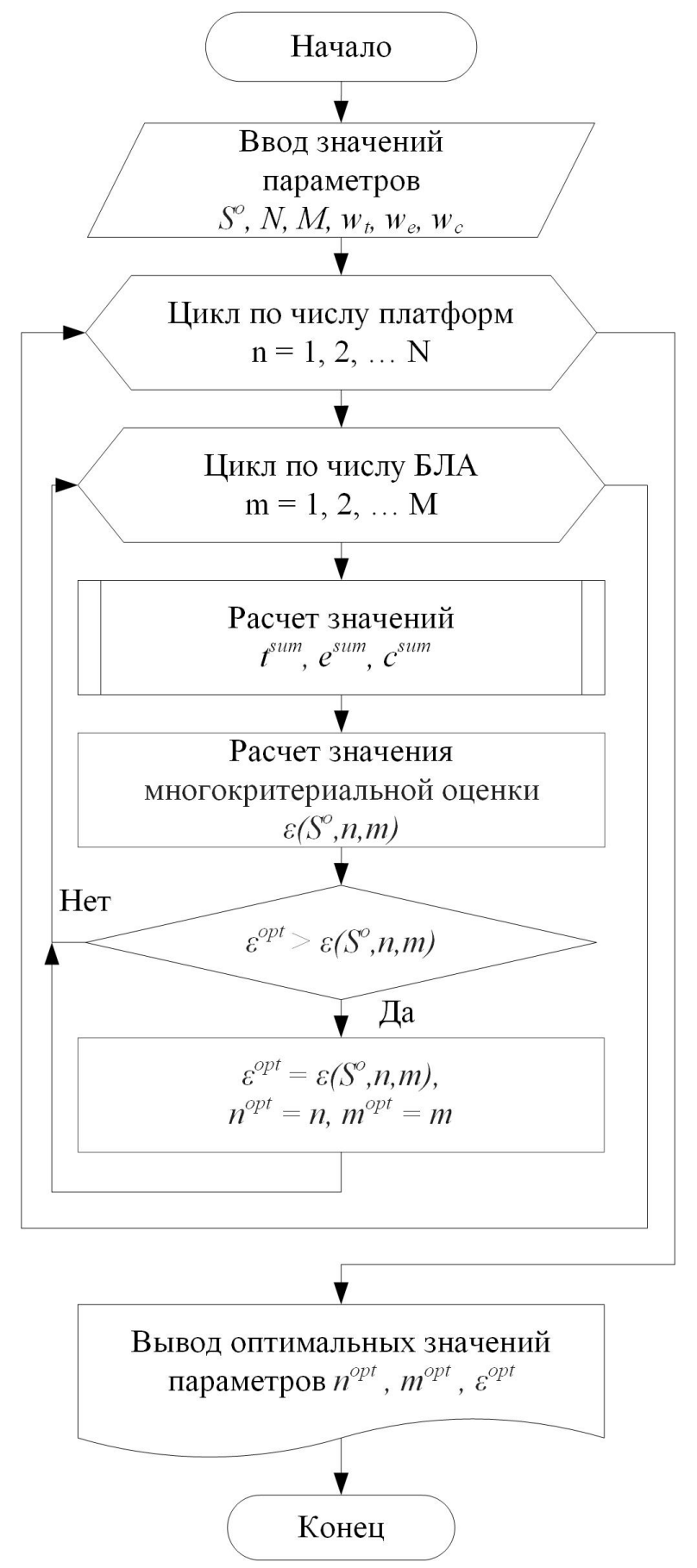

Рис. 1. Алгоритм расчета и выбора оптимальных значений количества обрабатывающей техники

ники на основе предложенного метода был разработан алгоритм, представленный на рис. 1.

При расчете суммарное время обработки $t^{\text {sum }}$ будем считать, что сервисные платформы и находящиеся на них БЛА передвигаются к месту обработки сельскохозяйственного угодья одновременно, поэтому основные временные затраты складываются из времени движения платформ к угодью и назад, а также времени обработки угодья $t^{0}$ и времени обслуживания БЛА $t^{u}$ :

$$
t^{\text {sum }}=2 t^{C P}+t^{o}+t^{u},
$$

где $t^{o}=\frac{S^{o}}{\Delta s n m}-$ среднее время работы каждого задействованного БЛА на сельскохозяйственном угодьи, число БЛА $n \in(0, N]$, число платформ $m \in(0, M]$. Для расчета времени 
обслуживания БЛА необходимо определить сколько раз за период $t^{o}$ потребуется произвести пополнение БЛА физическими и энергетическими ресурсами:

$$
t^{u}=\frac{t^{o}}{r_{\max }^{U} \Delta t}\left(2 t^{P S}+t_{P}^{u}\right)+\frac{t^{o}}{e_{\max }^{U} \Delta t}\left(2 t^{P S}+t_{P}^{u}\right),
$$

тогда:

$$
\begin{aligned}
& t^{\tilde{s i n}}=2 t+\frac{S^{o}}{\Delta s n m}+\frac{\frac{S^{o}}{\Delta s q s m}}{r_{\max }^{U} \Delta t}(2 t+ \\
& \left.+t_{P}^{u}\right)+\frac{\frac{S^{o}}{\Delta s n m}}{e_{\max }^{U} \Delta t}\left(2 t^{P S}+t_{P}^{u}\right)= \\
& =2 * t^{\tilde{N} P}+\frac{S^{o}}{\Delta s n m}+\frac{S^{o}}{\Delta s n m r_{\max }^{U} \Delta t}\left(2 t^{P S}+\right. \\
& \left.+t_{P}^{u}\right)+\frac{S^{o}}{\Delta s n m e_{\max }^{U} \Delta t}\left(2 t^{P S}+t_{P}^{u}\right) .
\end{aligned}
$$

При расчете суммарных энергетических ресурсов, необходимых для обработки сельскохозяйственного угодья $O$, также следует учесть передвижение платформ от центральной базы и обратно, а также обслуживание аккумуляторов всех БЛА в процессе работы:

$$
\begin{aligned}
e^{s u m}= & n 2 e_{\min }^{P}+\frac{S^{o}}{\Delta s n m e_{\max }^{U} \Delta t} n m= \\
= & n 2 e_{\min }^{P}+\frac{S^{o}}{\Delta s e_{\max }^{U} \Delta t} .
\end{aligned}
$$

Суммарная стоимость задействованной техники будет складываться из стоимости платформ и БЛА, обслуживаемых на них:

$$
c^{\text {sum }}=n c^{P}+m c^{U} .
$$

Для минимизации влияния конечных условий была произведена модификация вышеприведенных формул. Дополнительно были введены следующие временные переменные:

$$
t_{U}^{r}=r_{\max }^{U} \frac{\Delta t}{\Delta r}-\text { время, когда заканчиваются }
$$
физические ресурсы БЛА;

$$
t_{U}^{e}=\left(e_{\max }^{U}-2 e_{\min }^{U}\right) \frac{\Delta t}{\Delta e}-\text { время, когда закан- }
$$
чивается энергетические ресурсы БЛА.

С учетом соотношения времени расходования физических и энергетических ресурсов, расчет оценок времени обработки угодья и затрачиваемой энергии изменился следующим образом:

$$
\begin{aligned}
& t^{\text {sum }}=2 t^{C P}+\frac{s_{\max }^{o_{j}} \Delta r}{n m r_{\max }^{U} \Delta t}\left(2 t^{P S}+t_{P}^{u}+\frac{r_{\max }^{U} \Delta t}{\Delta r}\right), \\
& e^{s u m}=n 2 e_{\min }^{P}+\frac{s_{\max }^{o_{j}} \Delta r}{r_{\max }^{U} \Delta t}\left(2 t^{P S}+\frac{r_{\max }^{U} \Delta t}{\Delta r}\right) \Delta e, \\
& \text { если } t_{U}^{r}<t_{U}^{e} \text {; } \\
& \left\{t^{\text {sum }}=2 t^{C P}+\frac{s_{\max }^{o_{j}} \Delta e}{n m\left(e_{\max }^{U}-2 e_{\min }^{U}\right) \Delta t}\left(t_{P}^{u}+\frac{e_{\max }^{U} \Delta t}{\Delta e}\right),\right. \\
& \begin{array}{c}
e^{\text {sum }}=n 2 e_{\min }^{P}+\frac{s_{\max }^{o_{j}} \Delta e}{\left(e_{\max }^{U}-2 e_{\min }^{U}\right) \Delta t} e_{\max }^{U} . \\
\text { иначе. }
\end{array}
\end{aligned}
$$

Если $t_{U}^{r}<t_{U}^{e}$, это означает, что физические ресурсы закончились раньше, чем энергетические ресурсы. В случае если $t_{U}^{r}=t_{U}^{e}$, то физические и энергетические ресурсы заканчиваются одновременно $r_{\max }^{U} \frac{\Delta t}{\Delta r}=\left(e_{\max }^{U}-2 e_{\min }^{U}\right) \frac{\Delta t}{\Delta e}$. Это позволяет увеличить коэффициент использования энергии для одного взлета, что сводит к минимуму общее количество взлетов для выполнения задания.

Многокритериальной оценкой, используемой при принятии решения о количестве необходимой техники для обработки сельскохозяйственного угодья, является линейная комбинация описанных выше критериев:

$$
\varepsilon\left(S^{o}, n, m\right)=t^{\text {sum }} w_{t}+e^{\text {sum }} w_{e}+c^{\text {sum }} w_{c},
$$

где $w_{t}, w_{e}, w_{c}$ весовые коэффициенты, отвечающие за приоритеты затрат по времени, энергии и стоимости соответственно. В результате сортировки значений многокритериальной оценки выбирается некоторый кортеж значений количества сервисных платформ и БЛА для заданной площади сельскохозяйственного угодья $\varepsilon^{\text {opt }}$, имеющий минимальную оценку:

$$
\underset{n \in N, m \in M}{\operatorname{Arg} \min }\left(\varepsilon\left(S^{o}, n, m\right)\right)=<n^{*}, m^{*}>.
$$

Значения весов выбираются экспертным путем. В наших экспериментах были использованы следующие значения:

$w_{t}=3, w_{e}=1, w_{c}=1$ - случае приоритета повышения скорости обработки;

$w_{t}=1, w_{e}=2, w_{c}=3$ - в случае приоритета сокращения стоимости обработки. 
Рекомендующая программная система оценивания состава гетерогенных робототехнических ...

\section{ОПИСАНИЕ \\ РЕКОМЕНДУЮЩЕЙ ПРОГРАММНОЙ СИСТЕMЫ AGROBOTMODELING}

Разработанные методы, алгоритмы и программные модули были использованы при разработке рекомендующей программной системы AgrobotModeling, обеспечивающей многокритериальную оценку оптимального количества БЛА и платформ, необходимой для обработки заданной площади угодья в процессе численного и имитационного моделирования [17-20]. Структура системы представлена на рис. 2, состоящая из ряда программных модулей и необходимых баз данных. Система реализована в виде исполняемого диалогового приложения ОC Windows с использованием программных средств и среды разработки Microsoft Visual Studio 2017 на языке $\mathrm{C} / \mathrm{C}++$.
Модуль ввода исходных данных позволяет вводить параметры, такие как общая площадь обрабатываемого угодья, количество БЛА и платформы, используемых в обработке угодья.

В блок численного моделирования включены модуль многокритериальной оценки, модуль вывода оптимального расчета результатов и модуль вывода результатов в файл данных. Модуль многокритериальной оценки производит численную обработку на основе входных параметров из исходных данных. Результаты расчета экспортируются в файл данных txt c со значениями входных и выходных параметров, а также выбранными оптимальными значениями, необходимых для поддержки принятия решения о количестве гетерогенных робототехнических комплексов.

Реализация имитационного моделирования в программном комплексе AgrobotMod-



Pис. 2. Общая структура рекомендующей программной системы AgrobotModeling 
eling осуществляется на основе трех модулей (модуль расчета параметров обрабатываемого угодья, модуль расчета энергетических ресурсов, модуль расчета физических ресурсов). Модуль расчета параметров обрабатываемого угодья рассчитывает текущие площади обрабатываемого угодья, которые были завершены БЛА. Модуль расчета энергетических ресурсов рассчитывает текущие объемы заряда аккумуляторов БЛА и текущие объемы энергетических ресурсов сервисных платформ для обслуживания аккумуляторов БЛА. Модуль расчета физических ресурсов рассчитывает текущие объемы контейнеров БЛА и текущие объемы физических ресурсов сервисных платформ для обслуживания аккумуляторов БЛА.

Модуль проверки состояния степени выполнения работ сравнивает текущую обработанную площадью сельскохозяйственного угодья с общей площадью обрабатываемого угодья. Модуль проверки энергетических ресурсов сравнивает текущие объемы зарядов аккумуляторов БЛА и текущие объемы энергетических ресурсов сервисных платформ для обслуживания аккумуляторов БЛА с минимальным/максимальным значением. Модуль проверки физических ресурсов сравнивает текущие объемы контейнеров БЛА и текущие объемы физических ресурсов сервисных платформ для обслуживания аккумуляторов БЛА с минимальным/максимальным значением.

Модуль отображения результатов моделирования позволяет вывести численные результаты моделирования на экран. Модуль визуализации состояния обрабатываемого угодья позволяет отобразить изменение текущей обработанной площади сельскохозяйственного угодья. Модуль визуализации состояния платформ отобразить изменение текущих объемов энергетических ресурсов и физических ресурсов сервисной платформы для обслуживания аккумуляторов БЛА.

Модуль визуализации состояния БЛА отобразить изменение состояния и текущих объемов заряда аккумулятора и контейнера БЛА.

\section{РЕЗУЛЬТАТЫ И ИХ ОБСУЖДЕНИЕ}

На рис. 3 показан пользовательский интерфейс разработанной программы AgroBotModeling, визуализирующей процесс моделирования обработки сельскохозяйственного угодья с различным числом платформ и БЛА. Интерфейс содержит 4 основных блока, отражающие параметры угодья, платформы и БЛА, а также элементы управления и индикации прошедшего времени.

Блок сельскохозяйственного угодья позволяет ввести начальные значения следующих параметров: 1) общая площадь обрабатываемого угодья; 2) площадь, обрабатываемая одним БЛА за $\Delta t$. Также в блоке отображается текущее значение обработанной площади угодья.

В блоке сервисных наземных платформ задаются начальные значения следующих параметров: 1) количество платформы, используемых в обработке угодья; 2) максимальный объем энергетических ресурсов, размещаемых на одной сервисной платформе; 3) максимальный объем физических ресурсов, размещаемых на одной сервисной платформе. Также отображаются текущие значения следующих параметров: 1) объем оставшихся энергетических ресурсов на сервисной платформе; 2) объем оставшихся физических ресурсов на сервисной платформе.

В блоке БЛА задаются начальные значения следующих параметров: 1) количество БЛА, транспортируемых на одной сервисной платформе; 2) максимальный объем заряда аккумулятора БЛА; 3) максимальный объем контейнера с физическими ресурсами на БЛА; 4) набор опций, влияющих на порядок функционирования БЛА. Также отображаются текущие значения следующих параметров: 1) оставшийся заряд аккумулятора БЛА; 2) оставшийся объем физических ресурсов на БЛА; 3) текущий режим функционирования БЛА. 
Рекомендующая программная система оценивания состава гетерогенных робототехнических ...

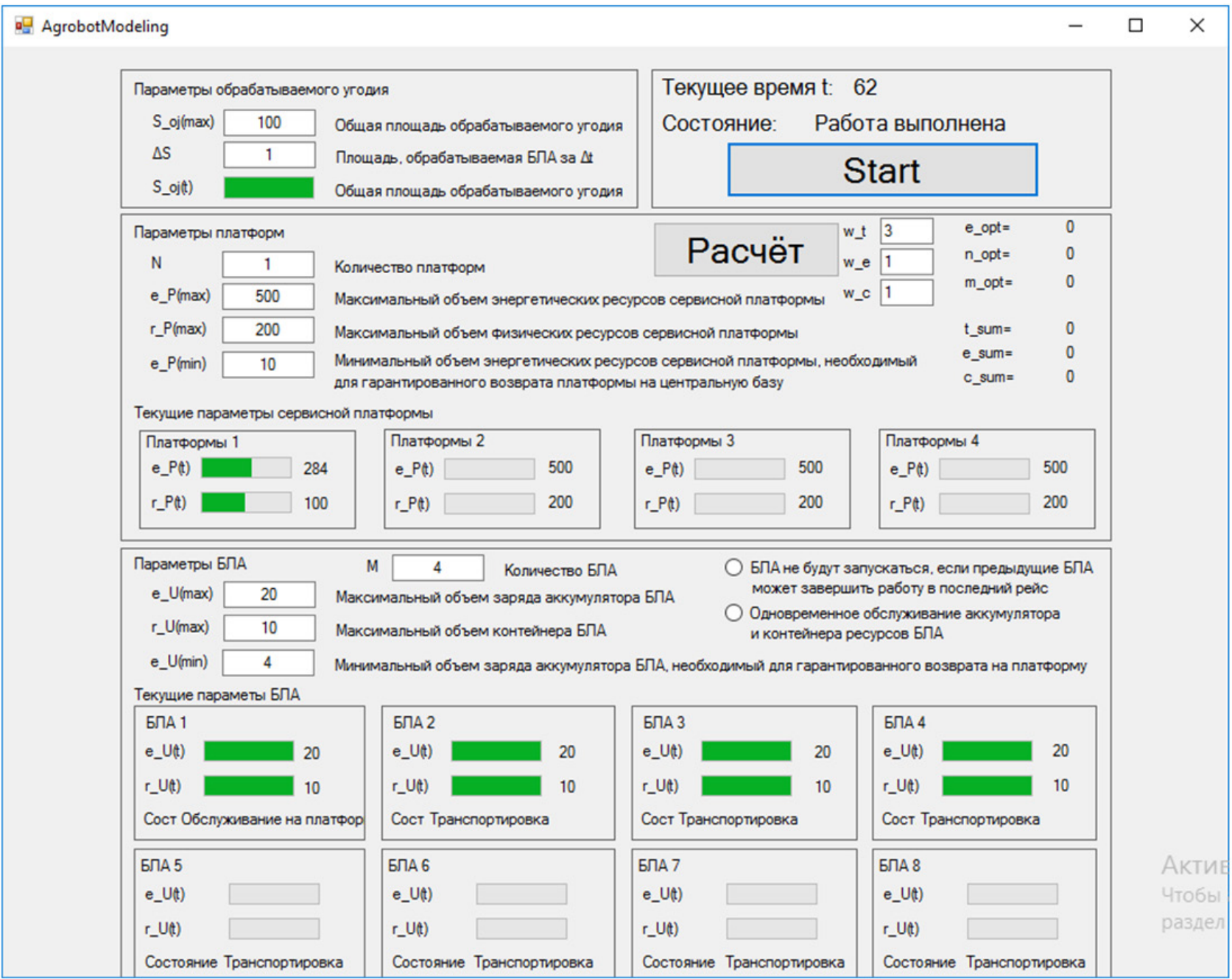

Рuс. 3. Пример пользовательского интерфейса программы AgroBotModeling

\section{ЗАКЛЮЧЕНИЕ}

Для оценивания необходимого состава и количества техники для обработки сельскохозяйственного угодья был разработан метод многокритериальной оценки с использованием линейной комбинации трех основных критериев суммарного время обработки, израсходованной энергии, стоимости задействованной техники, где весовые коэффициенты, отвечающие за приоритеты затрат по времени, энергии и стоимости выбираются экспертным путем.

Разработанные метод и алгоритм были использованы при создании рекомендующей программной системы AgrobotModeling, обеспечивающей в процессе численного и имитационного моделирования многокритериальную оценку оптимального количества
БЛА и наземных сервисных платформ, необходимого для обработки заданной площади угодья. Система реализована в виде исполняемого диалогового приложения OC Windows с использованием программных средств и среды разработки Microsoft Visual Studio 2017 на языке $\mathrm{C} / \mathrm{C}++$.

\section{СПИСОК ЛИТЕРАТУРЫ}

1. Андреев, В. П. Метод информационного взаимодействия для систем распределённого управления в роботах с модульной архитектурой / В. П. Андреев, П. Ф. Плетенев // Труды СПИИРАН. - 2018. - № 57 (2). - С. 134-160.

2. Лавренов, А. В. Особенности обработки данных и знаний об эпизодах социально-значимого поведения в окрестности интервью / А. В. Лавренов, А. В. Суворова, А. Е. Пащенко, 
А. Л. Тулупьев // Труды СПИИРАН. - 1. № 15 (4). - C. 246-262.

3. Ватаманюк, И. В. Моделирование траекторий перемещения робототехнических комплексов при реконфигурации пространственного положения роя / И. В. Ватаманюк, Г. Ю. Панина, А. Л. Ронжин // Робототехника и техническая кибернетика. - 2015. - № 3(8). C. 52-57.

4. Фетисов, В. С. Сравнительная характеристика автоматических зарядных и обменно-зарядных станций для обслуживания малых электрических беспилотных летательных аппаратов / В. С. Фетисов, Ш. Р. Ахмеров // Авиакосмическое приборостроение. - 2019. № 2. - С. 3-10.

5. Башилов, А. М. Автономные беспилотные летательные аппараты в точных системах агропроизводства / А. М. Башилов, В. А. Королев // Вестник аграрной науки Дона. 2018. - T. 3, № 43. - C. 76-82.

6. $V u, Q$. Trends in Development of UAVUGV Cooperation Approaches in Precision Agriculture / Q. Vu, M. Raković, V. Delic, A. Ronzhin // International Conference on Interactive Collaborative Robotics. Springer. LNCS. - 2018. - Vol. 11097. - P. 213-221.

7. Годжаев, 3. А. Развитие работ по созданию робототехники сельхозназначения / 3. А. Годжаев, А. П. Гришин, И. А. Пехальский, А. А. Гришин, В. А. Гришин // Политематический сетевой электронный научный журнал Кубанского государственного аграрного университета. - 2016. - № 119. - С. 488-502.

8. Yakovlev, R. Iterative method for solving the inverse kinematics problem of multi-link robotic systems with rotational joints / R. Yakovlev, A. Denisov, R. Prakapovich // Proceedings of 14th International Conference on Electromechanics and Robotics "Zavalishin's Readings". 2019. - P. 237-251.

9. Krestovnikov, K. Wireless Power Transmission System Based on Coreless Coils for Resource Reallocation Within Robot Group / K. Krestovnikov, E. Cherskikh, P. Smirnov // International Conference on Interactive Collaborative Robotics. - Springer, LNAI 11659. - 2019. C. 193-203.
10. Башилов, А. М. Аэромобильная агротехнология стимуляции роста и развития растений в агротехноценозах / А. М. Башилов, В. А. Королев // АгроСнабФорум. - 2017. № 8 (156). - C. 64-67.

11. Pavliuk, N. Development of Multipurpose Mobile Platform with a Modular Structure / N. Pavliuk, I. Kharkov, E. Zimuldinov, V. Saprychev // Proceedings of 14th International Conference on Electromechanics and Robotics "Zavalishin's Readings". - 2019. - P. 137-147.

12. Kemper, P. F. UAV Consumable Replenishment: Design Concepts for Automated Service Stations / P. F. Kemper, K. A. O. Suzuki, J. R. Morrison // Journal of Intelligent \& Robotic Systems. - 2011. - Vol. 61. - P. 369-397.

13. Maini, $P$. On cooperation between a fuel constrained UAV and a refueling UGV for large scale mapping applications / P. Maini, P. B Sujit // In: 2015 International Conference on Unmanned Aircraft Systems (ICUAS). - P. 1370-1377.

14. Zhang, K. Dynamic operations and pricing of electric unmanned aerial vehicle systems and power networks / K. Zhang, L. Lu, C. Lei, H. Zhu, Y. Ouyang // Transportation Research Part C: Emerging Technologies. - 2018. - 92. P. 472-485. - URL: https://doi.org/10.1016/j. trc.2018.05.011.

15. Daniel, C. Gandolfo. Energy evaluation of low-level control in UAVs powered by lithium polymer battery / Daniel C. Gandolfo, Lucio R. Salinas, Mario E. Serrano, Juan M. Toibero // ISA Transactions. - 2017. - Volume 71, Part 2. P. 563-572. - URL: https://doi.org/10.1016/j.isatra.2017.08.010.

16. Tao, LEI. State of art on energy management strategy for hybrid-powered unmanned aerial vehicle / Tao LEI, Zhou YANG, Zicun LIN, Xiaobin ZHANG // Chinese Journal of Aeronautics. - 2019. - Volume 32, Issue 6. P. 1488-1503. - URL: https://doi.org/10.1016/j. cja.2019.03.013.

17. Нго, К. Т. Анализ подвижных роботизированных платформ для обслуживания аккумуляторов беспилотных летательных аппаратов / К. Т. Нго, О. Я. Соленая, А. Л. Ронжин // Труды МАИ. - 2017. - № 95. - URL: http:// trudymai.ru/published.php? $\mathrm{ID}=84444$ 
Рекомендующая программная система оценивания состава гетерогенных робототехнических ...

18. Нго, К. Т. Функциональная модель взаимодействия БЛА с наземной роботизированной платформой при решении сельскохозяйственных задач / К. Т. Нго, В. В. Нгуен, И. Ю. Харьков, Е. Е. Усина, О. О. Шумская // Известия Кабардино-Балкарского научного центра РАН. - 2018. - №6-3 (86). - С. 41-50.

19. Соленая, О. Я. Анализ требований и ограничений мобильных электромеханических систем обслуживания аккумулято- ров беспилотных летательных аппаратов / О. Я. Соленая, К. Т. Нго, А. Л. Ронжин // Вопросы электромеханики. Труды ВНИИЭМ. 2017. - Том 159, № 4. - C. 42-48.

20. Denisov, Alexander. Mathematical and Algorithmic Model for Local Navigation of Mobile Platform and UAV Using Radio Beacons / Alexander Denisov, Roman Iakovlev, Igor Lebedev. // Interactive Collaborative Robotics (ICR2019). - 2019. - LNAI 11659. - P. 53-62.

Нго Куок Тьен - аспирант, Санкт-Петербургский институт информатики и автоматизации Российской академии наук. E-mail: quoctienbn@gmail.com.

Нгуен Ван Винь - аспирант, Санкт-Петербургский государственный университет аэрокосмического приборостроения. E-mail: nguyenvanvinhhvkt@gmail.com.

Ронжин Андрей Леонидович - д-р техн. наук, профессор, Санкт-Петербургский институт информатики и автоматизации Российской академии наук, Санкт-Петербургский государственный университет аэрокосмического приборостроения.

E-mail: ronzhin@iias.spb.su

\title{
RECOMMENDING SOFTWARE SYSTEM FOR ESTIMATING THE COMPOSITION OF HETEROGENEOUS ROBOTS FOR PERFORMANCE OF AGRICULTURAL OPERATIONS
}

\begin{abstract}
Q. T. Ngo ${ }^{\star}$, V. V. Nguyen ${ }^{\star *}$, A. L. Ronzhin ${ }^{\star \star * *}$
${ }^{*}$ St. Petersburg Institute for Informatics and Automation of the Russian Academy of Sciences

Annotation. An analytical review of the work aimed at increasing the battery life of UAV s and their maintenance on service ground robotic platforms, including in the field of agricultural robotics, is given. Moreover, the number of involved robots depends on the size of the cultivated area, the required speed of the task and other factors. Therefore, the aim of this study was to develop the formal approach and software for recommending system that provides an assessment of the composition of heterogeneous robotic tools for agricultural operations. The method of multicriteria estimation of the quantity and composition of heterogeneous agricultural robotic complexes required for processing a given area of a field, weather conditions and other aspects affecting the cost and speed of work is described. The recommending AgrobotModeling software system used to modeling and visualization of the interaction process of several ground service platforms and unmanned aerial vehicles during the processing of agricultural objects is presented. The user interface, containing 4 main blocks, reflecting the parameters of the land, platform and UAVs, as well as controls and indicators of elapsed time, provides visualization and modeling of the processing of agricultural land with a different number of platforms and UAVs.

Keywords: UAV, robotic service platform, farmland processing, UAV service, interaction of heterogeneous robots.
\end{abstract}

Ngo Quoc Tien - Postgraduate student of the St. Petersburg Institute for Informatics and Automation of the Russian Academy of Sciences. E-mail: quoctienbn@gmail.com

Nguyen Van Vinh - Postgraduate student of the St. Petersburg State University of Aerospace Instrumentation. E-mail: nguyenvanvinhhvkt@gmail.com

Ronzhin Andrey Leonidovich - Doctor of technical sciences, Professor of the St. Petersburg Institute for Informatics and Automation of the Russian Academy of Sciences and the St. Petersburg State University of Aerospace Instrumentation. E-mail: ronzhin@iias.spb.su 